\title{
Underwater image transmission system based on visible light communication
}

\author{
Zhou Yang, Wang Jinpeng, Guan Xin, Zhang Teng, Li Bo, Zou Nianyu \\ Dalian Polytechnic University, 116034, Dalian, China \\ wangjpedlpu.edu.cn
}

\begin{abstract}
In order to improve the image transmission quality of underwater visible light communication, this paper designs an optimal scheme of the underwater image transmission system based on blue visible light communication. In the hardware circuit module, the trans-impedance amplifier circuit of the T-feedback network is used to improve the signal receiving performance. Due to the bad feature of the underwater channel, a fast likelihood blind detecting algorithm was adopted to detect, collect and process the data in this article. The method proposed in the paper is able to reduce BER (Bit Error Rate) as well as speed up computation. It can be seen from the experimental results that the scheme of the system optimization effectively decreases DC bias of the photoelectric receiver, as well as decreases the computation complexity and improves the transmitting efficiency under the condition of meeting the requirements for the specific BER of the receiving signal.
\end{abstract}

Keywords: Underwater visible light communication, Image transmission, Detection algorithm.

\section{Introduction}

The ocean occupies about $71 \%$ of the earth's surface area, and it plays a significant role in the aspects of national security, economy and environment etc. The communication technique, a means for exchanging information, can be a key, which is the basic and supporting power for production activities in different fields of the marine environment. With the rapid and stable development of the modern society, there are more and more marine production activities, consequently, human being has a higher demand to the communication techniques. The transmissions of underwater image data has been widely and deeply applied and researched in many fields, such as exploration of deep-sea resources, monitoring of seabed ecological conditions, underwater rescue, ocean war preparation, and development of intelligent underwater robots [1-4]. At the same time, as the support technology of underwater image transmission, the research on underwater wireless communication technology is also expanding. Currently, the traditional underwater wireless communication technologies mainly include underwater acoustic communication, underwater radio frequency communication and underwater visible light communication. Underwater acoustic communication technology has low data transmission rate and slow 
propagation speed of sound waves, so there is a high communication delay. In addition, sound waves will also affect the safety of marine life; The communication distance of underwater radio frequency communication technology is extremely short, usually only a few meters, and the communication cost is high. In 2016, the experimental results of the research team of Lloret $\mathrm{J}$ et al. showed that radio frequency signals can be transmitted at a rate of up to $100 \mathrm{Mbps}$, but the transmission distance is extremely short, and the effective communication distance is only about $15 \mathrm{~cm}[5]$; Compared with the above two methods, the underwater visible light communication technology uses blue-green visible light with a wavelength of $450 \mathrm{~nm}$ $550 \mathrm{~nm}$ for data transmission in seawater, which has a very high data transmission rate, low link delay and low implementation cost [6-8]. On November 13, 2020, the "Struggle" manned submersible and the deep-sea video lander "Canghai" and "Lingyun" carried out joint operations in the Mariana Trench, among which the "Struggle" and the "Canghai" realized information exchange through visible light communication technology, and implemented the world's first live TV broadcast on the ocean floor of 10,000 meters [9].

The underwater channels are harsh, and many factors such as the optical properties, absorption and scattering effects of seawater will cause interference to signal transmission and affect communication quality. In order to solve the problem of low efficiency of traditional underwater wireless communication methods and improve the transmission effect of visible light communication, this paper proposes an optimized scheme of underwater image transmission system based on blue visible light communication. Firstly, the paper presents a system model for image transmission using blue visible light communication Secondly, in order to solve the problem of weak light signal the receiving end uses the T-feedback network trans-impedance amplifier circuit to improve system performance. According to the transmission characteristics of the underwater channel, the fast likelihood detection algorithm is used to optimize the detection of the optical signal to improve the accuracy and speed of the received signal. Finally, the feasibility of the system is verified by analyzing the experimental results. The results show that, compared with the traditional underwater communication technology, The scheme of this paper is stable and reliable, and has practical significance for improving the safety and scientific of underwater activities.

\section{System design principle and scheme}

The underwater image transmission system is mainly composed of three parts: transmitter, underwater channel and receiver, as shown in Figure 1. At the transmitter end part, after the collected image information is encoded and modulated by the MCU, the modulated signal is modulated into the current of the LED drive circuit and emitted into the underwater channel in the form of blue visible light. The signal passing through the underwater channel is received at the signal receiving end, and a photodetector is used to convert the optical signal received from the transmitting end into an electrical signal. The signal is extracted by the fast likelihood blind detection 
algorithm, and then the electrical signal is amplified by the operational amplifier circuit. The amplified electrical signal is demodulated and A/D converted to restore the original image data.

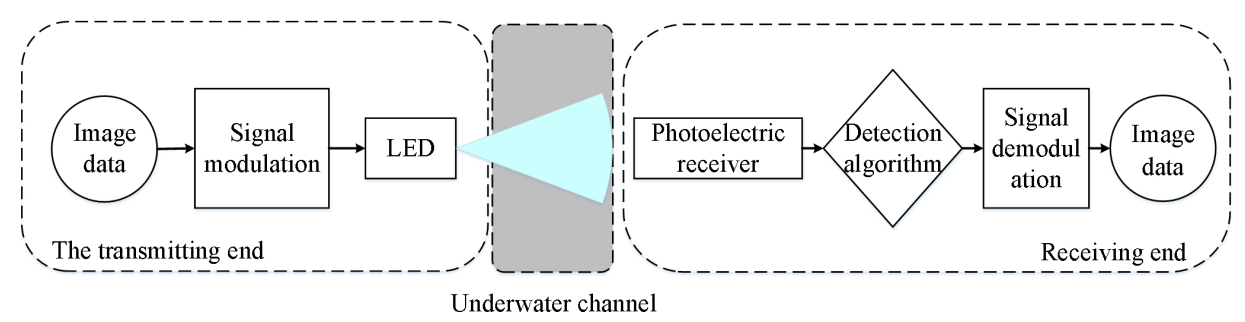

Fig. 1. System block diagram

\section{System hardware design}

\subsection{Transmitter circuit design}

After the image data is modulated, the transmitter drives the LED light source to convert electrical signals into optical signals for transmission. The blue LED drive circuit first uses an operational amplifier to form a voltage comparator to solve the problem of insufficient transistor drive capability. The function of the capacitor $\mathrm{C} 1$ is to increase the switching rate of the LED drive circuit, so that the high frequency performance of the drive circuit is improved. The effect of R2 is to balance the impedance at the input and the voltage. R3 and R4 protect the circuit and prevent short circuit to the ground. The schematic diagram of the drive circuit is shown in Figure 2.

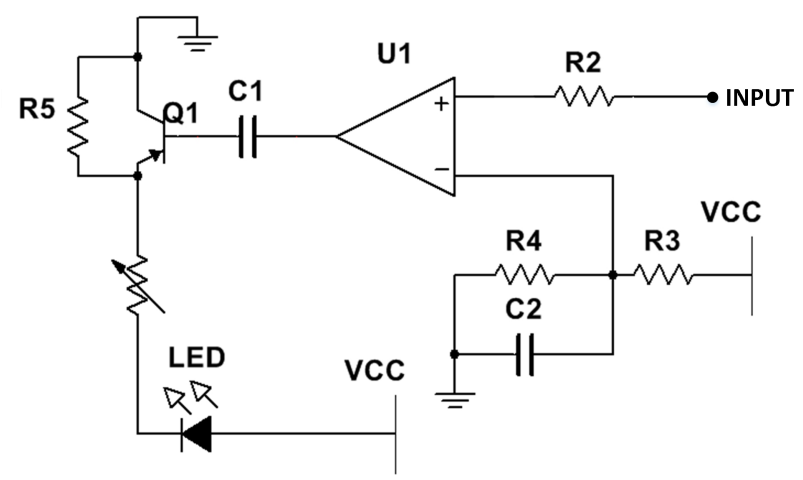

Fig. 2. Schematic diagram of drive circuit 


\subsection{Receiver circuit design}

Since the signal generated by the photodiode is relatively weak, the receiving end generally uses an operational amplifier circuit to amplify the received signal. When the gain of the ordinary operational amplifier circuit is very large, the resistance of the feedback resistor and the matching resistor will also become very large. After the input bias current of the amplifier flows through the matching resistor, a significant voltage is applied to the photodiode. However, due to the sensitive nature of the sensor will produce a larger dark current, which will produce a DC bias at the output, which will affect the circuit performance. In order to ensure the quality of signal transmission and improve the gain level of visible light signals, the trans-impedance amplifier circuit designed by the T-feedback network is introduced at the receiving end. The schematic diagram of the trans-impedance amplifier circuit of the Tfeedback network is shown in Figure 3.

In the embodiment, the circuit uses compensation resistor R5 to produce offset components caused by the input current of the operational amplifier, and its resistance value is the same as Rf. C2 is a bypass capacitor matched with the resistor R5 to reduce the noise introduced by the matched resistor R5. The T-feedback network is used to replace a single large-value feedback resistor, where R2 $\gg \mathrm{R} 3 \gg \mathrm{R} 1$, The equivalent impedance formula of the T-feedback network is:

$$
R_{\text {feq }}=R_{2}+R_{3}+\frac{R_{2} R_{3}}{R_{1}} \approx\left(1+\frac{R_{3}}{R_{1}}\right) R_{2}
$$

The trans-impedance amplifier circuit of the T-feedback network used a smaller resistor R2 to replace the original larger feedback impedance, but achieved the same current gain. The resistance of the compensation resistor decreased with the decrease of the new feedback resistor R2, and the corresponding voltage applied to the two segments of the photodiode decreases, the dark current decreased, and the matching error between the feedback resistor and the compensation resistor is reduced.

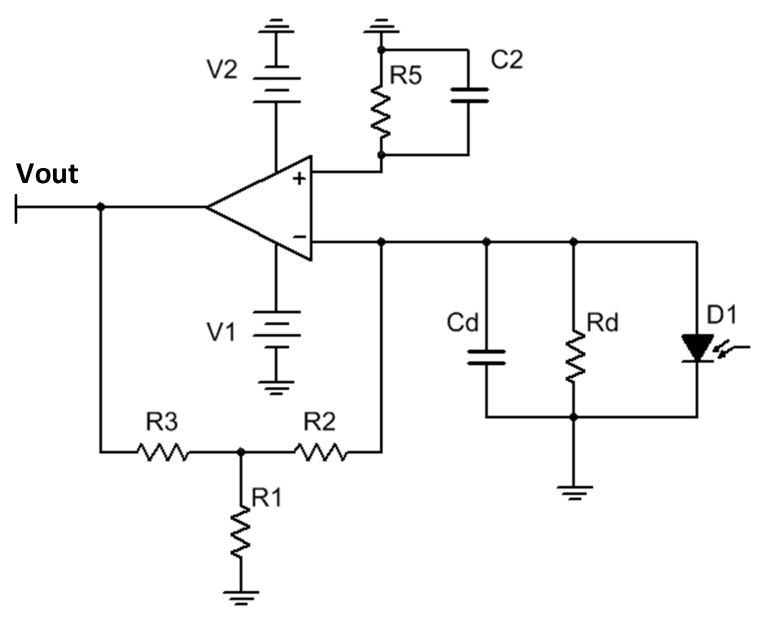

Fig. 3. Receiver circuit schematic diagram 


\section{System software part design}

\subsection{Signal modulation method}

CAP (Carrier-Free Amplitude / Phase Modulation) modulation technology is based on the development of QAM (Quadrature Amplitude Modulation) technology. Its characteristic is that shaping filters and matched filters are used at the transmitting end and the receiving end to generate and separate the same direction and quadrature signals, so that the generated CAP signal has no carrier in the signal band, so this modulation method is called carrier-free modulation[10]. Since the visible light communication system is very sensitive to system non-linearity, CAP modulation will not cause the system PAPR (Peak to Average Power Ratio) to be too high. This is also the advantage of cap modulation in optical communication system[11]. The CAP modulation principle block diagram is shown in Figure 4.

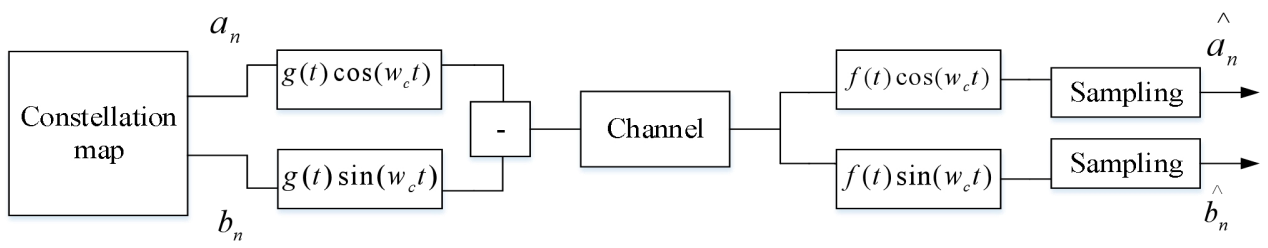

Fig. 4. CAP modulation principle diagram

\subsection{Fast-likelihood blind detection algorithm}

The indoor visible light channel communication distance is short and the channel is stable. However, the underwater visible light channel is different from the indoor visible light channel. Due to the harsh underwater environment, impurities and particles in the water, turbulence effects, water temperature changes, absorption and scattering effects of seawater and many other factors will interfere with the state of the underwater visible light channel. Therefore, the performance of the underwater visible light communication system is affected. For the transmission characteristics of underwater visible light channel, it is very important to select an appropriate signal detection algorithm.

Blind likelihood detection means that the receiver processes signal under the condition that the mathematical model and statistical parameters of the channel are not known, then it can complete the accurate estimation of the channel and the detection of the signal. Compared with the traditional MMSE (Minimum Mean Square Error) algorithm [12]、MD (Mean Detection) algorithm[13-14] and blind detection algorithm, the fast likelihood blind detection algorithm has lower complexity and higher accuracy. When the channel conditions such as channel model and parameters are unknown, the fast likelihood blind detection algorithm can complete the signal detection and channel model estimation by processing the received signal. 
The visible light channel function obeys the logarithmic normal distribution [1516], and the probability density function of the received signal $\mathrm{r}$ with respect to the transmitted signal $\mathrm{S}$ is:

$$
f_{x}(r \mid s, G)=\frac{1}{\left(\sqrt{2 \pi \sigma_{n}^{2}}\right)^{L}} \exp \left(-\frac{\left\|r-P_{s} G s\right\|^{2}}{2 \sigma_{n}^{2}}\right)
$$

Where $\mathrm{G}$ is the channel gain, is the transmission power, and $\mathrm{L}$ is the data length. According to the maximum likelihood decoding criterion, the sequence that makes the probability density function the maximum can be obtained by formula (2):

$$
\hat{s}=\arg \max f_{x}(r \mid s, G)
$$

Where $\hat{S}$ is the transmitted signal sequence, and formula (3) can also be understood as finding the estimate value of the signal that minimizes the Euclidean distance between $\mathrm{s}$ and $\mathrm{r}$ at the data set:

$$
\hat{s}=\arg \min \|r-G s\|^{2}
$$

Visible light communication has a high amount of data transmission, and it has a high complexity when searching and calculating according to formula (4). the complexity is $2^{\mathrm{L}}$. Now simplify it, expand and merge the above formula to obtain:

$$
\left\|r-P_{s} G s\right\|^{2}=\sum_{i=1}^{L} r_{i}^{2}-2 P_{s} G \sum_{i=1}^{L} r_{i} s_{i}+P_{s}^{2} G^{2} \sum_{i=1}^{L} s_{i}^{2}
$$

According to the non-negative characteristics of the visible light channel and the analysis of the signal value only " 0 " and " 1 ", in order to determine the number of bits "1" in the transmitted signal $\mathrm{s}$, the square sum function of the signal is defined as:

$$
S=\sum_{i=1}^{L} s_{i}^{2}, 0 \leq S \leq L
$$

At the same time, set the symbol corresponding to bit "1" to be the largest, sort the received signals and find the sum of the first $\mathrm{S}$ items:

$$
Q_{S}=r_{(1)}+r_{(2)}+\ldots+r_{(s)},\left(r_{(1)} \geq r_{(2)} \ldots \geq r_{(S)}, 1 \leq S \leq L\right)
$$

Substituting formula (5) and formula (6) into formula (4), simplification can be obtained:

$$
\left\|r-P_{s} G s\right\|^{2}=\sum_{i=1}^{L} r_{i}^{2}-2 P_{s} G Q_{S}+P_{s}^{2} G^{2} S
$$

Formula (7) can get the decision criterion of the signal sum function $\mathbf{S}$ through extreme value analysis:

$$
\hat{S}=\left\{\begin{array}{l}
\underset{1 \leq S \leq L}{\arg \max } \frac{Q_{S}^{2}}{S}, Q_{S}>0 \\
0, Q_{S}<0
\end{array}\right.
$$


According to the judgment criterion of the signal sum function $\mathbf{S}$, the received signal $r_{(i)}$ can be judged. The data order judgment condition of the receiving end is:

$$
\mathrm{r}_{(i)}=\left\{\begin{array}{l}
1, i \geq \hat{S} \\
0, i \leq \hat{S}
\end{array}\right.
$$

The first relative maximum in the decision function (9) is the absolute maximum of a complete signal transmission. The image of this function is approximately a convex function, so the signal can be demodulated through the first peak of the decision function. This decision method Further reduce the complexity of calculation.

\section{$5 \quad$ Experiment and analysis}

\subsection{Experimental simulation analysis}

The T-feedback network trans-impedance amplifier circuit at the photoelectric receiving end was simulated, and compared with the ordinary trans-impedance amplifier circuit, the input current signal frequency was a square wave signal of $10 \mathrm{kHz}$, and the simulation results are shown in the figure 5. The simulation results show that the bias current generated by the T-feedback network trans-impedance amplifier circuit is significantly reduced. Compared with the ordinary transimpedance amplifier circuit, the DC bias is reduced by $55.9 \%$. The T-feedback network can effectively reduce the DC bias by the compensation resistor. 


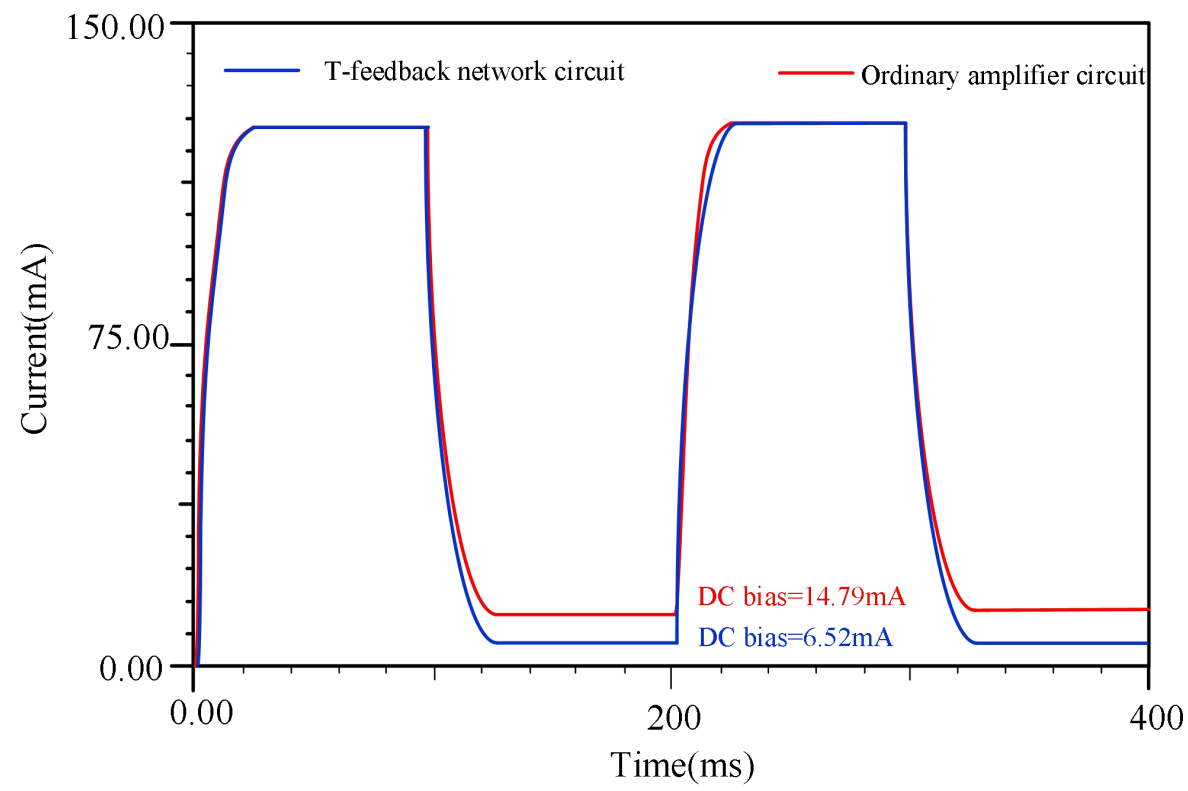

Fig. 5. Simulation diagram of output waveform comparison

For the performance analysis of the algorithm, under the conditions of data length $\mathrm{L}=20$, and variance $=\sigma_{n}^{2}=0.5$, the simulation results of the BER performance of the Fast-likelihood blind detection algorithm, the MMSE algorithm and the mean detection algorithm are shown in Figure 6.

It can be seen that when the same BER of $10^{-4}$ is reached, the SNR(Signal-Noise Ratio) of the Fast-likelihood blind detection algorithm is about 28dB, and the SNR of the MMSE algorithm is about $36 \mathrm{~dB}$. In contrast, the BER is reduced by $22 \%$. Obviously the fast-likelihood blind detection algorithm is better than other algorithms. In addition, each time the detection is performed, the detection time of the Fast likelihood blind detection algorithm is $3.4 \mathrm{~s}$, the Mean detection algorithm is $5.2 \mathrm{~s}$, and the MMSE algorithm is $6.8 \mathrm{~s}$. It can be seen that the algorithm used in this article not only met the need for BER performance, but also reduced the computational complexity and improves the communication rate. It has advantages in the high-speed signal transmission of the visible light communication system. 


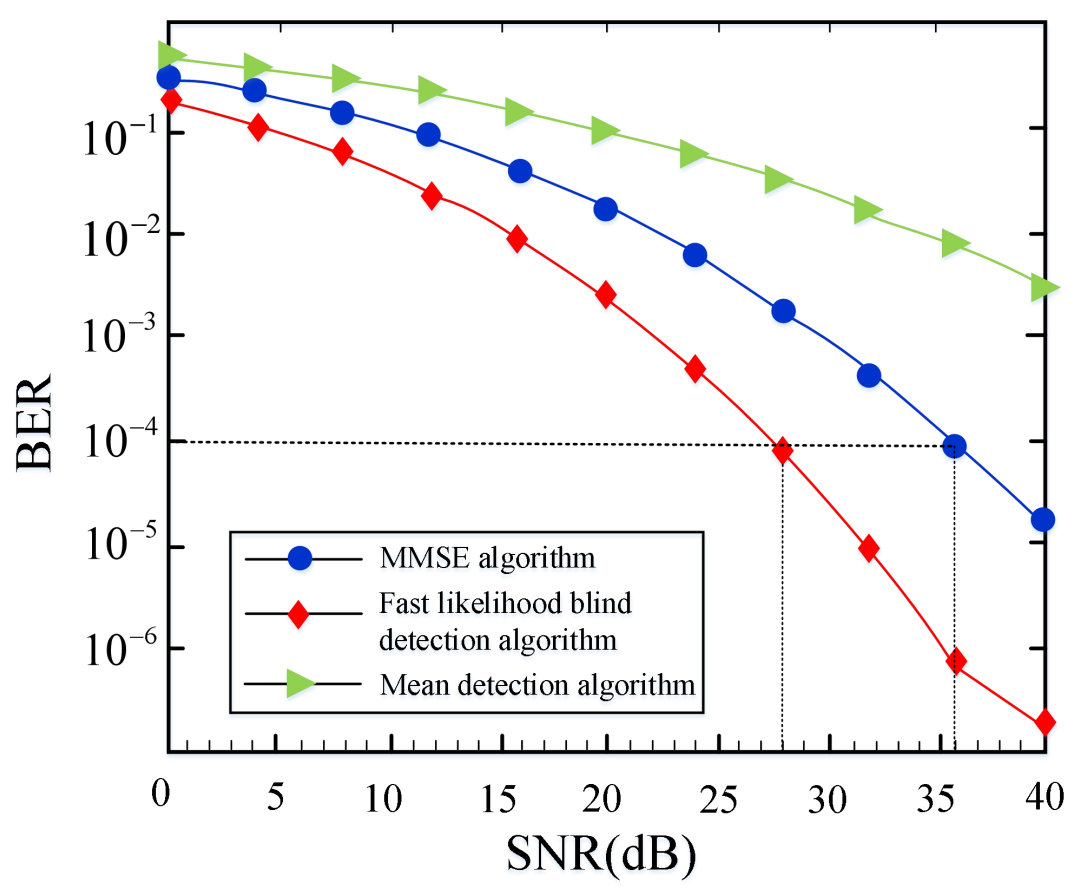

Fig. 6. BER diagram of the three algorithms

\subsection{Image transmission test}

Figure 7 is the system test diagram built indoors. A water tank with a specification of $100 \mathrm{~cm} * 30 \mathrm{~cm}^{*} 15 \mathrm{~cm}$ is used to simulate the underwater channel. During the experiment, adjust the angle of the LED light source at the transmitting end and the angle of the photodiode at the receiving end to align them to achieve the best transmission effect. Figure 8 shows the BER under different transmission distances.

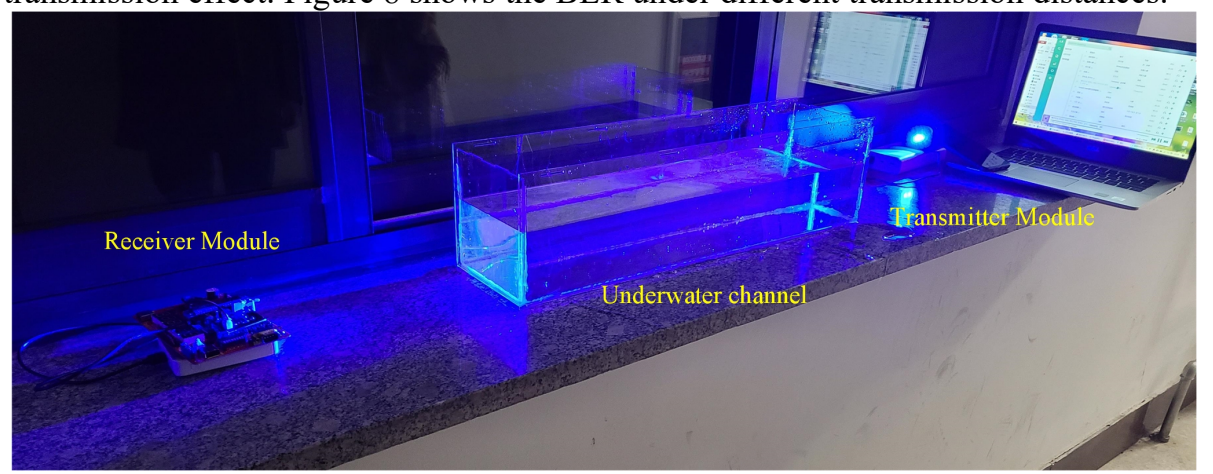

Fig. 7. System experiment diagram 


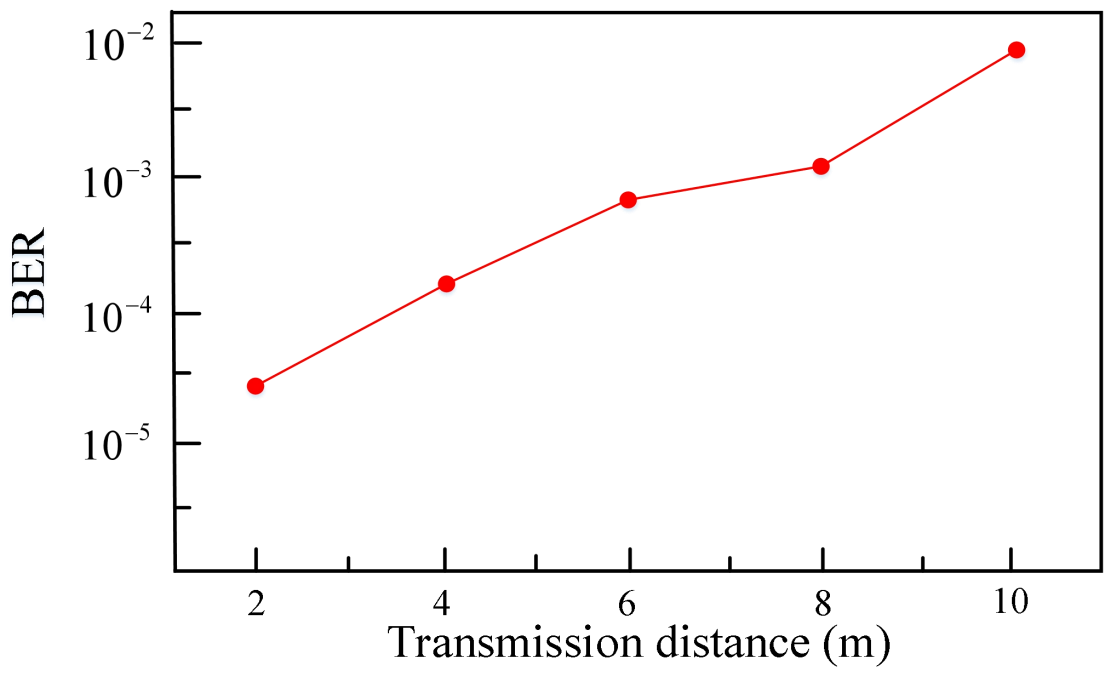

Fig. 8. BER under different transmission distances

In order to better verify the effectiveness of the scheme and simulate underwater image transmission, the sent images are now divided into three types of underwater green, blue and yellow backgrounds. The image size is $380 * 380$ pixels. Figure 9 is the transmitted image, and Figure 10 is the received image. It can be seen from the figure that the three received images are almost the same as the original images, and only some details are blurred.

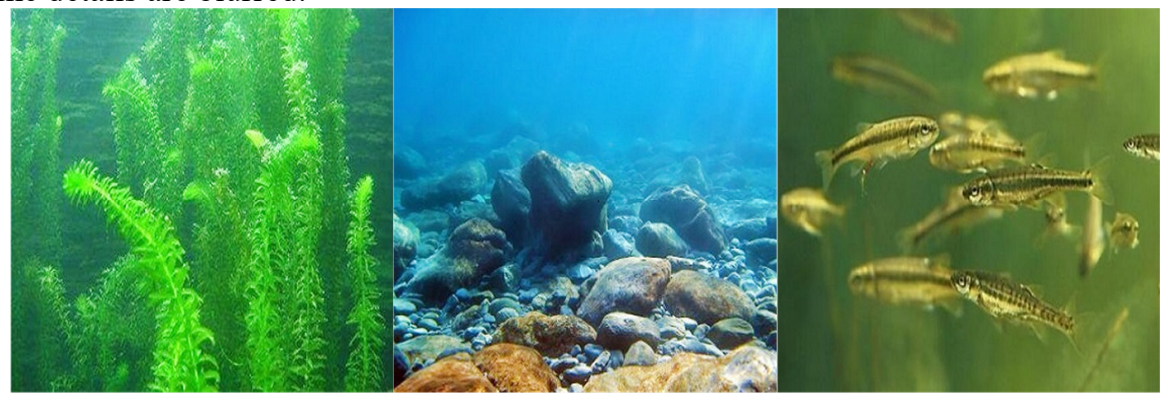

Fig. 9. The transmitted image 


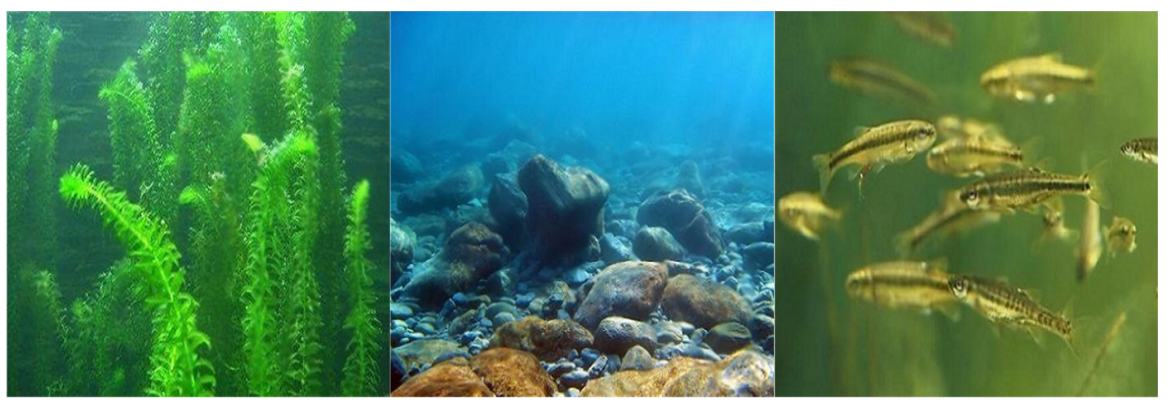

Fig. 10. The received image

\subsection{Objective analysis of image quality}

In the objective evaluation part, the UCIQE(Underwater Color Image Quality Evaluation) and MSE(Mean-square Error) are used to objectively analyze the received image quality. UCIQE is a linear combination of color concentration, saturation and contrast, which is used to evaluate the color deviation, blurriness and low contrast of underwater images. The higher the value, the higher the image quality. MSE can evaluate the degree of image change. The smaller the value of MSE, the higher the accuracy of the image. The experimental results are shown in Table 1.

Table 1. Comparison of objective evaluation of different methods

\begin{tabular}{cccccccc}
\hline & \multicolumn{2}{c}{ Green image } & \multicolumn{2}{c}{ Blue image } & \multicolumn{2}{c}{ Yellow image } \\
\cline { 2 - 8 } & UCIQE & MSE & UCIQE & MSE & UCIQE & MSE \\
\hline $\begin{array}{c}\text { Conventional circuit } \\
\text { and MD algorithm } \\
\begin{array}{c}\text { T-circuit and MD } \\
\text { algorithm }\end{array}\end{array}$ & 0.5328 & 2563.8 & 0.5278 & 3418.2 & 0.5331 & 2633.0 \\
$\begin{array}{c}\text { Conventional circuit } \\
\text { and MMSE algorithm } \\
\begin{array}{c}\text { T-circuit and MMSE } \\
\text { algorithm }\end{array}\end{array}$ & 0.6814 & 1150.0 & 0.6742 & 1089.3 & 0.6625 & 1201.4 \\
$\begin{array}{c}\text { This article scheme } \\
\text { (n) }\end{array}$ & 0.7341 & 863.7 & 0.7584 & 811.3 & 0.7528 & 763.5 \\
\hline
\end{tabular}

It can be seen from Table 1 that the evaluation index of UCIQE of the scheme in this paper is obviously higher than that of other schemes. This is because the dark current of the conventional receiving circuit is large and the signal transmission effect is poor; The MD algorithm algorithm has certain requirements on the data length, and the judgment error is larger when the length is different. In the changing underwater light environment, the MMSE algorithm is not sensitive to the light intensity, which 
leads to large errors. There are two reasons for the better image reception of our scheme: 1) The trans-impedance amplifier circuit of the T-feedback network used reduces the dark current of the circuit while ensuring the circuit gain remains unchanged, and at the same time reduces the matching error between the resistors, the circuit performance is better; 2) The Fast-likelihood blind detection algorithm used in this paper filters out edge noise through constrained search, does not rely on channel statistical characteristics, reduces the amount of calculation, and improves the detection accuracy. The scheme in this paper has a higher degree of restoration of the original image's color density, saturation, and contrast.

In addition, the MSE index of the scheme in this paper is also significantly lower than other schemes, indicating that the pixel gap between the received image and the original image is smaller, and the image information fidelity is better.

\section{Conclusion}

Aiming at the disadvantages of traditional underwater communication methods and the poor underwater visible light communication channels, this paper designed a visible light communication system for underwater image transmission. The transimpedance amplifier circuit of the T-feedback network was adopted at the receiving end of the system to improve the performance of signal transmission; The use of the Fast-likelihood blind detection algorithm not only reduced the BER of signal transmission, but also reduced the computational complexity. Finally, the effectiveness of the proposed scheme was verified by building a system experiment platform and comparing the image quality under different schemes.

Acknowledgements: This research was financially supported via Project of the National Natural Science Foundation of China (61402069), the 2017 Project of the Natural Science Foundation of Liaoning province (20170540059), the General project of Liaoning education department in 2016(2016J205).

\section{References}

1. Lu, H., et al. : CO-Net: A cognitive ocean network. IEEE Wireless Com-mun. 26(3), 90 $96(2019)$

2. Wang J P, Ye Z P, Tarun M, Li B and Zou N Y.: A Novel Linear Antenna Synthesis for Linear Dispersion Codes Based on an Innovative HYBRID Genetic Algorithm, Symmetry 2019,11(9),1176.

3. Xiang W D, Yang P, Wang S, et al. : Underwater image enhancement based on red channel weighted compensation and gamma correction model [J]. Opto-Electron Adv 1, 2018, 01(10): 180024.

4. Zeng $\mathrm{F}$ J,Yang $\mathrm{K}$ J,Yan X,Zhao M M,Yang Ping,Wen L H.: Research progress of underwater laser communication system [J/OL]. Laser and Optoelectronics Progress:119[2020-12-21].

5. Wang J P, Ye Z P, Jeremy Gillbanks, Tarun M. Sanders and Zou N Y, A Power Control Algorithm Based on Chicken Game Theory in Multi-Hop Networks, Symmetry 2019, 
$11(5), 718$

6. ZHAO Y H, WANG C, WANG F M.: $1.725 \mathrm{~Gb} / \mathrm{s}$ underwater visible light communication system based on a silicon substrate green LED and equal gain combination receiver [C] // International Conference and Exhibition on Visible Light Communications (Invited Talk 3). Yokohama, Japan: ICEVLC, (2018).

7. Wang J P, Cao F, Zou N Y. Multi carrier system joint receiving method based on MAI and ICI. [J], Jilin Daxue Xuebao (Gongxueban), 2018, 41(6): 301-305.

8. CHI N, WANG C F, LI W P.: Research Progress of Underwater Visible Light Communication Technology Based on Blue/Green LED. Journal of Fudan University(Natural Science), 58(05), 537-548 (2019).

9. Chen H H.: "Struggle": From domestic to "National Innovation". China Science News, 2020-11-30 (1).

10. ZOU P, LIU YF, WANGF M, CHIN, Mitigating nonlinearity characteristics of graycoding square 8QAM in underwater VLC system [C] / Asia Communications and Photonics Conference. Hangzhou, China: IEEE, 2018: 1-3.

11. Wang J P, Ye Z P, Cao F, and Zou N Y. : Cooperative distributed antenna transmission method based on co-channel interference in $5 \mathrm{G}$ mobile communication system, Journal of Jilin University (Engineering and Technology Edition), 2020, 48 (1): 333-341.

12. Ji W Y, Wu G F, Wang C. : Generalized Likelihood Block Detection for Spad-based Underwater Vlc System[J]. Science Letter, 2020.

13. Wang Y, et al. : Fusion-based underwater image enhancement by wavelet decomposition. In: Proceedings of IEEE International Conference on Industrial Technology (ICIT), Toronto, Canada, pp. 1013-1018 (2017)

14. Qi Y, Liu Z, Ni X L. : Research on Maximum Likelihood Detection Algorithm of Random Optical Signal Based on Expectation Maximization[J]. Journal of Changchun University of Science and Technology (Natural Science Edition), 2017, 40(02): 90-93.

15. Asuncion A, Newman D. UCI Machine Learning Repository[EB/OL]. [2015-06-01]. http://archive.ics.uci.edu/ml/datasets.html

16. Yu J J, Chi N. Digital Signal Processing In High-Speed Optical Fiber Communication Principle and Application[M]. Singapore, 2020 